\title{
Nachtrag zum Aufsatz: \\ Hypothesen und Beobachtungen über die Bedeutung der vertikalen Planktonwanderung.
}

Von

Dr, G. Burckhardt (Basel).

Meine Beobachtungsreihe vom Oktober 1909 aus dem Silsersee ist in einer Hinsicht nicht ganz einwandsfrei: Sie stammt aus der Nähe des Ausflusses, und es läßt sich behaupten, was ich dort beobachtet habe, sei lediglich die Flucht der Planktozoen vor dem Ausfluß, die durch Woltere $\mathrm{ck}^{1}$ ) bekannt geworden ist. Ich habe daher am 20. April 1910 an einer geeigneten Stelle im Genfersee (Léman) ähnliche Beobachtungen angestellt.

Der östliche Teil des Sees besitzt, wie alle Alpenrandseen, sehr steile Böschungen. Doch findet sich in der Mitte zwischen Vevey und Montreux bei la Maladaire eine unterseeische Terrasse nur etwa $20 \mathrm{~m}$ unter dem Spiegel. Sie erstreckt sich dem Ufer nach von den Roches de Salagnons bis gegen die Roche des Mouettes $1,2 \mathrm{~km}$ weit in einer Breite von 300 bis $600 \mathrm{~m}$. Ihr Rand erhebt sich dicht am Steilabfall an einer Stelle bis $18 \mathrm{~m}$ unter den Seespiegel (Punkt $357 \mathrm{im}$ schweizerischen topographischen Atlas) $440 \mathrm{~m}$ vom nächsten Ufer entfernt. An dieser Terrasse münden nur unbedeutende Bächlein, während im 0 . in 1,2 und $3 \mathrm{~km}$, im W. in $3,5 \mathrm{~km}$ Entfernung sich Mündungen ziemlich bedeutender, wilder Flüsse finden. Auch ist dieser Teil des Ufers noch relativ schwach bewohnt.

Der 18. und der Vormittag des 19. April waren ganz hell; bei der ziemlich großen Transparenz des Wassers (etwa diø Hälfte des winterlichen Maximums) wird das Zooplankton damals ziemlich tief gewandert sein. Nach meiner Hypothese wird es sich also auch vom Ufer weit entfernt haben. Am 20. April war der Himmel den ganzen Tag über mit einer gleichmäßigen Wolkenschicht bedeckt (vielstündiger Regen). Es blieb also am Beobachtungstag ein gröBerer Teil des Planktons an der Oberfläche. Nach meinen Annahmen mußten sich nun an dem trüben Tage mehr Zooplanktonten über dem tiefen Wasser finden, in das sie sich tags zuvor zurückgezogen hatten, als über der Terrasse, von der alles tiefer als $20 \mathrm{~m}$ Sinkende wegwandert:

1) Diese Zeitschrift, Bd. I, S. 303. 
Ich machte mit dem erwähnten Entomostrakennetz drei Vertikalfänge aus $18 \mathrm{~m}$ Tiefe bis zur Oberfläche über 18, 70, $130 \mathrm{~m}$ Tiefe, den ersten an der beschriebenen Stelle am Rand der Terrasse, den zweiten über ihrer Böschung nur $100 \mathrm{~m}$ weiter auben, den dritten über dem als Rhonedelta sanft gegen SSO. ansteigenden Boden in $800 \mathrm{~m}$ Uferferne, näher der nächsten Flußmündung und dem nächsten Dorfe. An den tiefern Lokalitäten machte ich außerdem noch tiefere Stufenfänge, von denen der tiefste $(67 \mathrm{~m})$ zur Not als Totalfang gelten kann.

Ich gebe die Resultate für die sicher begrenzten Zählkategorien in Form kleiner Utbersichten gesondert:

\begin{tabular}{|c|c|c|c|c|c|c|c|c|c|}
\hline \multirow{2}{*}{$\begin{array}{l}\text { Erklärung: } \\
\text { Tiefe des Sees } \\
\text { Uferdistanz } \\
\text { Tiefe des Fanges }\end{array}$} & \multirow{2}{*}{\begin{tabular}{|l|}
130 \\
800 \\
$\mathbf{1 5 ~ m}$ \\
$\mathbf{3 6 m}$ \\
$\mathbf{6 7} \mathrm{m}$ \\
\end{tabular}} & \multirow{2}{*}{$\begin{array}{r}70 \\
540 \\
\mathbf{1 8 ~} \mathbf{m} \\
\mathbf{3 6 m}\end{array}$} & \multicolumn{2}{|c|}{$\begin{array}{r}18 \mathrm{~m} \\
440 \mathrm{~m}\end{array}$} & & & & & \\
\hline & & & $18 \mathrm{~m}$ & $\mid \begin{array}{l}0 \\
0 \\
67 . \\
67-m- \\
\text { Fang }\end{array}$ & & & & & \\
\hline \multicolumn{10}{|l|}{ Resultate: } \\
\hline $\begin{array}{l}\text { Diaptomus gra- } \\
\text { cilis } \sigma^{\pi}\end{array}$ & $\begin{array}{l}260 \\
415 \\
415\end{array}$ & $\begin{array}{l}205 \\
260\end{array}$ & 65 & 15 & $\begin{array}{l}\text { Diaptomus gra- } \\
\text { cilis } ?\end{array}$ & $\begin{array}{l}245 \\
485 \\
485\end{array}$ & $\begin{array}{l}145 \\
200\end{array}$ & 45 & $9^{1 / 2}$ \\
\hline $\begin{array}{l}\text { Diaptomus laci- } \\
\text { niatus, } \\
\text { Erwachsene }\end{array}$ & $\begin{array}{l}12 \\
13 \\
24\end{array}$ & $\begin{array}{l}1 \\
4\end{array}$ & 0 & $\mathbf{0}$ & $\begin{array}{l}\text { Diaptomus gra- } \\
\text { cilis u. lacinia- } \\
\text { tus, Gesamtz. }\end{array}$ & $\begin{array}{l}1070 \\
1560 \\
1655\end{array}$ & $\begin{array}{l}5800 \\
820\end{array}$ & 200 & 12 \\
\hline $\begin{array}{l}\text { Cyclops strenu- } \\
\text { us ơ }\end{array}$ & $\begin{array}{l}24 \\
67 \\
67\end{array}$ & $\begin{array}{l}10 \\
15\end{array}$ & 0 & $\mathbf{0}$ & $\begin{array}{l}\text { Cyclops leuck- } \\
\text { arti ơ }\end{array}$ & $\begin{array}{r}2 \\
4 \\
15\end{array}$ & $\begin{array}{l}5 \\
5\end{array}$ & 1 & 7 \\
\hline $\begin{array}{l}\text { Cyclopsstrenuus } \\
\text { u. leuckarti, } \\
\text { Gesamtzahl }\end{array}$ & $\begin{array}{l}360 \\
555 \\
750\end{array}$ & $\begin{array}{l}150 \\
250\end{array}$ & 160 & 22 & $\begin{array}{l}\text { Bythotrephes } \\
\text { longimanus, } \\
\text { nur Junge }\end{array}$ & $\begin{array}{l}1 \\
1 \\
0\end{array}$ & $\begin{array}{l}0 \\
0\end{array}$ & 0 & 0 \\
\hline $\begin{array}{l}\text { Leptodora hyali- } \\
\text { na, nur Junge } \\
\text { vorhanden }\end{array}$ & $\begin{array}{l}4 \\
5 \\
4\end{array}$ & $\frac{2}{1}$ & 1 & 25 & $\begin{array}{l}\text { Sida limnetica, } \\
\text { nur Junge }\end{array}$ & $\begin{array}{l}0 \\
1 \\
0\end{array}$ & $\begin{array}{l}1 \\
2\end{array}$ & 0 & 0 \\
\hline $\begin{array}{l}\text { Bosmina core- } \\
\text { goni, Erwach- } \\
\text { sene }\end{array}$ & $\begin{array}{l}140 \\
525 \\
700\end{array}$ & $\begin{array}{r}42 \\
130\end{array}$ & 21 & 3 & $\begin{array}{l}\text { Bosmina core- } \\
\text { goni, Junge }\end{array}$ & $\begin{array}{r}60 \\
360 \\
520\end{array}$ & $\begin{array}{r}50 \\
115\end{array}$ & 54 & 10 \\
\hline \multirow[t]{2}{*}{$\begin{array}{l}\text { Bosmina core- } \\
\text { goni, Gesamt- } \\
\text { zahl }\end{array}$} & $\begin{array}{r}200 \\
88 \overline{5} \\
1220 \\
\end{array}$ & $\begin{array}{r}92 \\
245\end{array}$ & 65 & $\mathbf{b}^{1 / 3}$ & $\begin{array}{c}\text { Daphnia longi- } \\
\text { spina hyalina, } \\
\text { Gesamtzahl }\end{array}$ & $\begin{array}{c}0 \\
\left.28^{1}\right) \\
30^{1}\end{array}$ & $\begin{array}{l}\left.1^{2}\right) \\
\left.3^{2}\right)\end{array}$ & $\left.2^{3}\right)$ & $6^{2} / 2$ \\
\hline & & & & & $\begin{array}{l}\text { Gesamtzahl aller } \\
\text { Entomostrak. } \\
\text { exkl. Nauplien }\end{array}$ & $\begin{array}{l}1635 \\
3035 \\
3660\end{array}$ & $\begin{array}{r}830 \\
1320\end{array}$ & 430 & $11^{3} / 4$ \\
\hline $\begin{array}{l}\text { 1) Zur Hälfte } \\
\text { 2) Zu }{ }^{3 / 4} \text { Jun } \\
\text { 3) Alles Jung }\end{array}$ & & & & & & & & & \\
\hline
\end{tabular}


Das Verhältnis des limnetischen Fanges zum gleich tiefen litoralen ist also

$\begin{array}{ccrc}\text { im Genfersee } & 18 \mathrm{~m} & \mathbf{4} & \text { Tag! } \\ \text { im Silsersee } & 10 \mathrm{~m} & \mathbf{2 0} & \text { Nacht! } \\ \text { do. } & 5 \mathrm{~m} & \mathbf{3 5} & " \\ \text { do. } & 2^{1} / \mathrm{m} & \mathbf{5 0 0} & "\end{array}$

Kleiner war es zu erwarten, erstlich wegen der größeren Fangtiefe, außerdem weil es sich um Tagfänge handelt. Nachts wäre der Litoralfang gleich, der limnetische viel reicher ausgefallen; das Verhältnis wäre wohl auf 8 gestiegen.

Betrachten wir die einzelnen Kategorien, so finden wir

maximale Uferflucht bei erwachsenen Diaptomus laciniatus und erwachsenen Daphnia hyalina;

sehr starke Uferflucht bei Cyclops strenuus of, erwachsenen Bosminen (die Zahlen für Bythotrephes longimanus und Sida limnetica sind zu klein um viel zu beweisen);

mittlere Uferflucht bei Cyclops leuckarti $\sigma^{\pi}$, jungen Bosminen, jungen Daphnien, jungen Diaptomus, Diaptomus gracilis;

minimale (doch absolut immer noch beträchtliche!) Uferflucht bei $\mathrm{Cy}-$ clops- + und Jungen und jungen Leptodoren.

Damit müßten wir die vertikale Verteilung an den vorhergehenden sonnigen Tagen vergleichen. Diese konnte ich stürmischen Wetters wegen nicht aufnehmen. Die Verteilung am Fangtag selbst dürfen wir natürlich nicht berücksichtigen, da wir nicht annehmen dürfen, daß alle Planktozoen auf den betreffenden Lichtwechsel gleich reagieren. Ich benutze daher, was ich über die vertikale Wanderung unter denselben Umständen im Vierwaldstättersee beobachtet habe.

Diaptomus laciniatus zeigt die vertikale Wanderung am allerstärksten; Daphnia hyalina verläßt mindestens die obersten $15 \mathrm{~m}$ (Junge nur $10 \mathrm{~m}$ ) vollständig.

Ganz leer an Bosminen wird dagegen die oberste Schicht nicht, doch sinkt die Hauptmasse auf ca. $25 \mathrm{~m}$.

Von Diaptomus gracilis wären $10-20 \%$, von jungen wenig mehr in den oberen $18 \mathrm{~m}$ zu erwarten, von Cyclops leuckarti $\sigma^{\top}$ die Hauptmasse gerade etwa an der Grenze.

Cyclops- 9 und Junge lassen immer Vertreter in den höheren Schichten zurück und wandern weniger tief als Diaptomus; die jungen. Leptodoren bleiben zum gröBeren Teil in den obern ea. $20 \mathrm{~m}$ zurück.

In allen diesen Fällen stimmen vertikale Wanderung und die hier beobachtete Uferflucht sehr wohl überein. Nur Cyclops strenuus $\sigma^{7}$ wandert im Vierwaldstättersee nicht so tief, daß sich eine so 
starke Uferflucht erklären ließe. Ich habe aber im Luganersee bei hellem Wetter und geringerer Transparenz im Oktober folgende Verteilung beobachtet:

$$
\begin{array}{cc}
0--10 \mathrm{~m} & 0,5 \\
10-20 \mathrm{~m} & 3 \\
20-287 \mathrm{~m} & 96,5
\end{array}
$$

Noch einen Blick auf den Fang über $70 \mathrm{~m}$ Tiefe. Bei tief wandernden Formen muß er mehr mit dem litoralen übereinstimmen (Diaptomus laciniatus, erwachsene Daphnien), bei nicht tief wandernden mehr mit dem limnetischen (junge Bosminen, Cyclops leuckarti). DaB dies letztere sich nicht noch mehr und deutlicher zeigt, ist wohl die ausgleichende Wirkung des Windes. Zufälligerweise hätten wir die Zone der Anreicherung treffen können; doch sind die einzigen in Betracht fallenden Zahlen zu klein, um ernstliche Schlüsse zu erlauben (Sida limnetica, Cyclops leuckarti $\sigma^{7}$ ).

Wolterecks und meine Beobachtungen werden endlich bestätigt durch eine Mitteilung, die ich Herrn Prof. Hans Bachmann in Luzern verdanke: Im Vierwaldstättersee auf der $1,5 \mathrm{~km}$ langen und bis $1,4 \mathrm{~km}$ breiten Luzerner Rhede, deren Tiefe von 2 bis $9 \mathrm{~m}$ schwankt, sind Planktonfänge in der Regel sehr arm an Tieren. Will der genannte Planktologe Entomostrakenmaterial sammeln, so ist er genötigt bis über Tribschen hinauszufahren, wo der Boden ziemlich steil von 25 auf $100 \mathrm{~m}$ Tiefe absinkt. 